\title{
4-Hydroxy-2-nonenal Alkylated and Peroxynitrite Nitrated Proteins Localize to the Fused Mitochondria in Malpighian Epithelial Cells of Type IV Collagen Drosophila Mutants
}

\author{
András A. Kiss, ${ }^{1}$ Nikoletta Popovics, ${ }^{1}$ Zsolt Boldogkői, ${ }^{1}$ \\ Katalin Csiszár, ${ }^{2}$ and Mátyás Mink ${ }^{1}$ \\ ${ }^{1}$ Institute of Medical Biology, University of Szeged, Somogyi B. U. 4, 6720 Szeged, Hungary \\ ${ }^{2}$ John A. Burns School of Medicine, University of Hawaii, 1960 East West Road, Honolulu, HI 96822, USA
}

Correspondence should be addressed to Mátyás Mink; mink@bio.u-szeged.hu

Received 4 September 2017; Revised 3 December 2017; Accepted 2 January 2018; Published 30 January 2018

Academic Editor: Daniela Grifoni

Copyright (C) 2018 András A. Kiss et al. This is an open access article distributed under the Creative Commons Attribution License, which permits unrestricted use, distribution, and reproduction in any medium, provided the original work is properly cited.

\begin{abstract}
Background. Human type IV collagenopathy is associated with mutations within the COL $4 A 1$ and to a less extent the COL4A2 genes. The proteins encoded by these genes form heterotrimers and are the highest molar ratio components of the ubiquitous basement membrane. The clinical manifestations of the COL4A1/A2 mutations are systemic affecting many tissues and organs among these kidneys. In order to uncover the cellular and biochemical alterations associated with aberrant type IV collagen, we have explored the phenotype of the Malpighian tubules, the secretory organ and insect kidney model, in col4al collagen gene mutants of the fruit fly Drosophila melanogaster. In Malpighian epithelial cells of col4a1 mutants, robust mitochondrial fusion indicated mutation-induced stress. Immunohistochemistry detected proteins nitrated by peroxynitrite that localized to the enlarged mitochondria and increased level of membrane peroxidation, assessed by the amount of proteins alkylated by 4-hydroxy-2-nonenal that similarly localized to the fused mitochondria. Nuclei within the Malpighian epithelium showed TUNEL-positivity suggesting cell degradation. The results demonstrated that col4a1 mutations affect the epithelia and, consequently, secretory function of the Malpighian tubules and provide mechanistic insight into col4al mutation-associated functional impairments not yet reported in human patients and in mouse models with mutant COL4A1.
\end{abstract}

\section{Introduction}

Basement membranes (BMs) are nanoscale sheets of extracellular matrices that play essential roles in multiple organs including muscle homeostasis, structures, and integrity of the dermal and ocular system, neuromuscular junctions, and blood filtration in the kidneys. The most abundant structural components of BMs include laminins, collagen IV, nidogens, perlecan, and agrin [1]. The ubiquitous mammalian BMs consist of heterotrimeric type IV collagens with $(\mathrm{COL} 4 \mathrm{Al})_{2}$ COL4Al composition. The clinical presentation of patients with COL4A1 mutation is systemic with numerous affected organs and tissues including the eyes, brain, the vascular system, skeletal muscles, and kidneys [2,3]. A distinct form of type IV collagenopathy, Hereditary Angiopathy, Nephropathy, Aneurysms, and Muscle Cramps (HANAC) syndrome, is caused by $\mathrm{N}$-terminal mutations within the COL4A1 gene. The renal manifestation of the same mutations in mouse models includes albuminuria, hematuria, glomerular cysts, and delays in glomerulogenesis and podocyte differentiation [4].

We have identified an allelic series of dominant, temperature-sensitive, antimorph mutations in the cognate col4al gene of the fruit fly, Drosophila melanogaster. The $\mathrm{col}_{4 a 1^{+/-}}$heterozygotes are viable and fertile at permissive temperature of $20^{\circ} \mathrm{C}$ but die at $29^{\circ} \mathrm{C}$. In these mutants, we 
have reported severe myopathy [5], tortuous BM, detachment of the gut epithelial and visceral muscle cells from the BM [6], intestinal dysfunction, overexpression of antimicrobial peptides, and excess synthesis of the oxidants hydrogen peroxide and peroxynitrite [7]. Peroxynitrite, by substituting the hydrogen atom by a nitro $\left(-\mathrm{NO}_{2}\right)$ group adjacent to the hydroxyl group on the aromatic ring of tyrosine, adversely impacts protein functions and can be detected as a speciesindependent antigene [8]. Peroxynitrite can also remove a hydrogen atom from polyunsaturated fatty acids resulting in the formation of aldehydes, conjugated dienes, and hydroperoxyradicals that trigger a free radical chain reaction and membrane lipid damage by lipoperoxidation [9].

The main product of membranous polyunsaturated fatty acid peroxidation is the reactive 4-hydroxy-2-nonenal, HNE [10]. The reactivity of HNE with proteins relies on Michael addiction and, by modifying histidine residues, generates alkyl-conjugated polypeptides also detectable as speciesindependent antigens [11]. As there is no direct laboratory test to estimate lipid peroxidation, measurements of HNE-conjugated protein levels currently serve as surrogates [12]. The bulk of peroxynitrite reacts rapidly with carbon dioxide, present at $\sim 1 \mathrm{mM}$ in cells, forming the unstable product nitrosoperoxycarbonate $\left(\mathrm{ONOOCO}_{2}^{-}\right)$, one-third of which decomposes into carbonate $\left(\mathrm{CO}^{-\bullet}\right)$ and $\mathrm{NO}_{2}{ }^{\bullet}$ radicals and two-thirds into the neutral $\mathrm{NO}_{3}{ }^{-}$and $\mathrm{CO}_{2}$ [13].

Insect Malpighian tubules serve as secretory organs. These renal tubules lack a vascular blood system and float freely in the hemocoel (blood-filled body cavity). The tubules are surrounded by BM and consist of two epithelial cell types, the metabolically active principal and the intercalated stellate cells [14]. The insect renal system is aglomerular, and urine is formed by active transport rather than by selective reabsorption of ultrafiltrate as in vertebrates. While the insect tubule system represents an intermediate towards the glomerular kidney, it fulfills the same basic functions of transport, excretion, and osmoregulation [15].

We have recently shown that the col4al Drosophila mutants develop stress fibers in their Malpighian cells and aberrantly express cell-surface integrin receptors [16]. In the present study, we have extended our research to address altered posttranslational protein modifications by peroxynitrite and 4-hydroxy-2-nonenal in the Malpighian tubules. The col4al mutants demonstrated heavy protein tyrosine nitration and protein-histidine alkylation that localized to the enlarged and fused mitochondria as signs of mitochondrial stress. HNE-protein adducts colocalized with the cytoplasmic membrane that was accompanied by cell degeneration in the tubules performing TUNEL-positivity, collectively suggesting that these aberrant processes are integral parts of col4a1associated pathology.

\section{Materials and Methods}

2.1. Maintenance of Drosophila Strains. Wild-type Oregon flies and col4a1 mutant stock with the DTS-L3 allele were maintained at $20^{\circ} \mathrm{C}$ and $29^{\circ} \mathrm{C}$ on yeast-cornmeal-sucroseagar food, consisting of nipagin to prevent fungal infection.
The mutant stocks were kept heterozygous over the CyRoi balancer chromosome. Malpighian tubules were removed under carbon dioxide anesthesia from adults that were grown at both permissive and restrictive temperature for 14 days. Dissected Malpighian tubules were fixed in 4\% paraformaldehyde dissolved in phosphate buffered saline (PBS) for $10 \mathrm{~min}$, washed three times in PBS, permeabilized for $5 \mathrm{~min}$ in $0,1 \%$ Triton X, dissolved in PBS, and washed three times in PBS. Blocking was achieved in 5\% BSA dissolved in PBS for 1 hour and washed three times in PBS.

2.2. Immunostaining and Antibodies. Nuclei in the dissected Malpighian tubules were counterstained by $1 \mu \mathrm{g} / \mathrm{ml} 4^{\prime}, 6$ diamino-2-phenylindole (DAPI) in $20 \mu \mathrm{l} \mathrm{PBS}, 12 \mathrm{~min}$ in dark. F-actin was stained by 1 unit Texas $\operatorname{Red}^{\mathrm{TM}}-\mathrm{X}$ Phalloidin (ThermoFisher) in $20 \mu \mathrm{l}$ PBS for $20 \mathrm{~min}$. A-Mannopyranosyl and a-glucopyranosyl residues as membrane markers were stained by Concanavalin A, Alexa Fluor ${ }^{\mathrm{TM}} 594$ Conjugate (ThermoFisher) in $20 \mu \mathrm{l}$ PBS for 1 hour. We used $1 \mu \mathrm{l}$ mouse monoclonal anti-3-nitrotyrosine [39B6] (Abcam) in $20 \mu \mathrm{l}$ PBS for 1 hour and stained 4-hydroxynonenal conjugate by $1 \mu \mathrm{l}$ mouse monoclonal anti-4-hydroxynonenal antibody (Abcam) in $20 \mu \mathrm{l}$ PBS for 1 hour. Primary mouse antibodies were visualized by $1 \mu \mathrm{l} \mathrm{F}\left(\mathrm{ab}^{\prime}\right)$ 2-Goat Anti-Mouse IgG $(\mathrm{H}$ + L) Cross Adsorbed Secondary Antibody conjugated with Alexa Fluor 488 (ThermoFisher) in $20 \mu \mathrm{l}$ PBS for 1 hour and $1 \mu \mathrm{l}$ Goat Anti-Mouse IgG $(\mathrm{H}+\mathrm{L})$ Cross Adsorbed Secondary Antibody, Alexa Fluor 350, in $20 \mu \mathrm{l}$ PBS for 1 hour. Mitochondria were visualized by the mitochondrially targeted EYFP (mito-GFP) following appropriate crosses [17].

2.3. Confocal Microscopy. Photomicrographs of the Malpighian tubules were generated by confocal laser scanning fluorescence microscopy (Olympus Life Science Europa $\mathrm{GmbH}$, Hamburg, Germany). Microscope configuration was the following: objective lens: UPLSAPO 60x (oil, NA: 1.35); sampling speed: $8 \mu \mathrm{s} /$ pixel; line averaging: $2 \mathrm{x}$; scanning mode: sequential unidirectional; excitation: $405 \mathrm{~nm}$ (DAPI), $543 \mathrm{~nm}$ (Texas Red), and $488 \mathrm{~nm}$ (Alexa Fluor 488); laser transmissivity: $7 \%$ were used for DAPI, $42 \%$ for Alexa Fluor 488 and $52 \%$ for Texas Red.

2.4. TUNEL-Labelling. Terminal deoxyribonucleotide transferase-mediated dUTP-fluorescein conjugate nick end labelling (TUNEL) was carried out by using the in situ cell death detection kit (Roche) as recommended. Embryos of mutant and control flies were incubated at $20^{\circ} \mathrm{C}$ or $29^{\circ} \mathrm{C}$ and L3-stage larvae collected. Nuclei in the Malpighian tubules were counterstained by $1 \mu \mathrm{g} / \mathrm{ml} 4^{\prime}, 6$-diamidino-2phenylindole (DAPI). Labellings were visualized by a HundWetzlar fluorescence microscope by using FITC or DAPI filters.

\section{Results}

3.1. Heavy Protein Nitration in col4a1 Mutants. We have previously demonstrated that the col4al mutant flies synthesize peroxynitrite at higher concentration as part of their antimicrobial immune response under restrictive conditions [7]. 


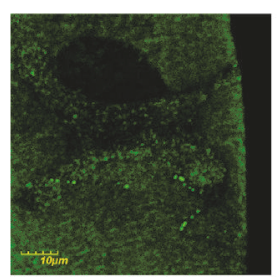

(a)

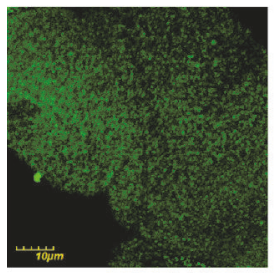

(g)

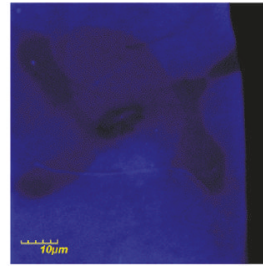

(b)

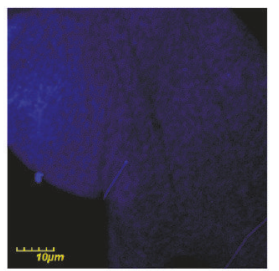

(h)

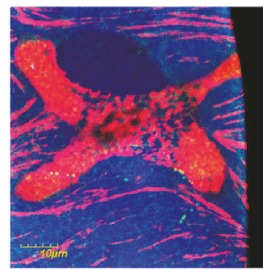

(c)

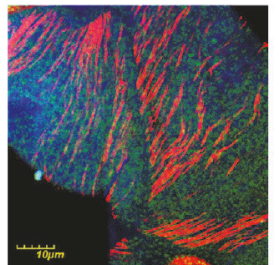

(i)

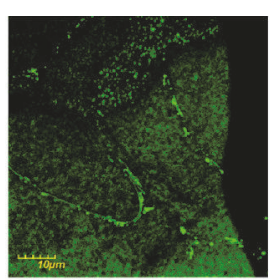

(d)

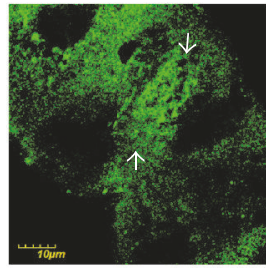

(j)

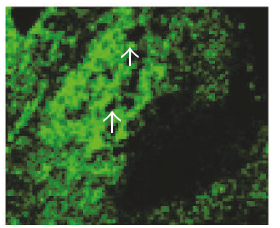

(m)

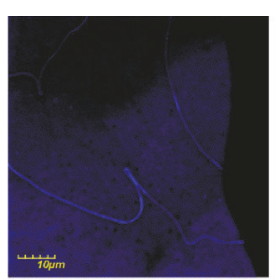

(e)

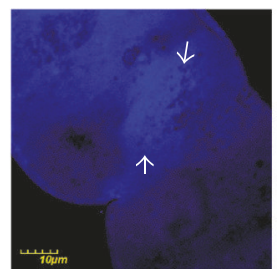

(k)

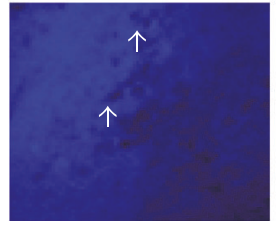

(n)

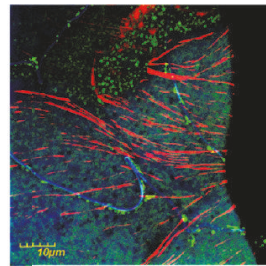

(f)

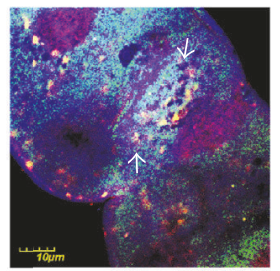

(l)

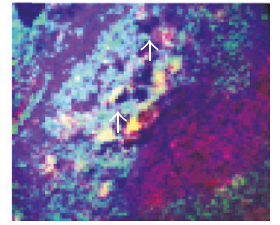

(o)

FIgURE 1: Protein nitration in Malpighian epithelial cells. Color code: mitochondria, green; nitrated proteins, blue; actin, red. (a) Wild-type flies incubated at $20^{\circ} \mathrm{C}$, mitochondria, (b) nitrated proteins, and (c) merge. Note a stellate cell in (c). ((d), (e), (f)) Wild-type flies incubated at $29^{\circ} \mathrm{C}$ and displayed in the same order. ((g)-(i)) Mutant flies, incubated at $20^{\circ} \mathrm{C}$, and $((\mathrm{j})-(\mathrm{l}))$ mutant flies, incubated at $29^{\circ} \mathrm{C}$. Photomicrographs are displayed in the same order as in the upper row. Localization of nitrated proteins to mitochondria is shown in (c), (i), (f), and (l). Uneven distribution and fusion of mitochondria are demonstrated in (j). White arrows in (j), (k), and (l) pointing the region displayed in higher magnification in $(\mathrm{m}),(\mathrm{n})$, and (o). White arrows in $(\mathrm{m}),(\mathrm{n})$, and (o) showing regions with no/few mitochondria and the lack of staining, demonstrating localization to mitochondria with nitrated proteins indirectly.

Peroxynitrite is produced by the diffusion-driven reaction of nitric oxide (NO) in the presence of oxidants such as the mitochondrial-derived radical superoxide anion, $\mathrm{O}_{2}{ }^{--}$. The sources of $\mathrm{NO}$ are at extramitochondrial sites and the dissolved gas diffuses into mitochondria, reacts with $\mathrm{O} 2^{\circ-}$, and disrupts protein functions by protein tyrosine nitration [18]. We therefore expected accumulation nitrated proteins in the mitochondria of col4al mutant flies following incubation at $29^{\circ} \mathrm{C}$.

We did not observe gross alterations in the Malpighian tubules of the mutants compared to control flies; mitochondria were distributed evenly in the cytoplasm and the fluorescent light intensities used to record nitrated proteins in the mutants were comparable to the control animals (Figures 1(a)-1(c) and Figures 1(g)-1(i)), following incubation at permissive condition. However, under restrictive conditions $\left(29^{\circ} \mathrm{C}\right)$, we noted marked differences in the Malpighian tubules of mutant flies. While mitochondria in the epithelial cells of wild-type Malpighian tubules remained evenly distributed with no shape alteration at this temperature (Figure 1(d)), in mutants, mitochondrial fusion and uneven distribution were observed (Figure 1(j)). The level of nitrated proteins was remarkably higher in mutants in comparison with the control (Figure $1(\mathrm{k})$ versus Figure $1(\mathrm{e})$ ) and these signals localized to the mitochondria (Figures 1(f) and 1(l)).

3.2. High Levels of Alkylated Proteins in the Mutants. The level of lipid peroxidation was determined indirectly by the accumulation of HNE-protein adducts. Results showed comparable amounts of alkylated proteins in the epithelial cells of mutant Malpighian tubules at permissive condition (Figure 2(b) versus Figure 2(h)), and the appearance of mitochondria remained unaffected in both mutants and controls under these conditions (Figure 2(a) versus Figure $2(\mathrm{~g}))$. In mutants under restrictive conditions $\left(29^{\circ} \mathrm{C}\right)$, uneven distribution and fusion of mitochondria occurred (Figure 2(j) versus Figure 2(d)), the mutants produced more HNE-protein adducts (Figure 2(k) versus Figure 2(e)), and the alkylated proteins localized to mitochondria (Figures $2(\mathrm{f})$ and 2(l)).

3.3. Protein-HNE Adducts Associate with Cytoplasmic Membrane. We next explored the involvement of the cytoplasmic membrane in col4al-associated pathology. We recorded numerous alkylation sites in form of punctate staining in colocalization with the cytoplasmic side of the membrane 


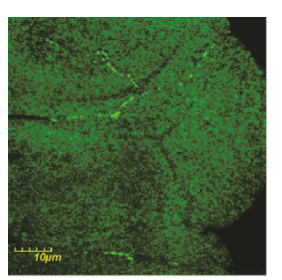

(a)

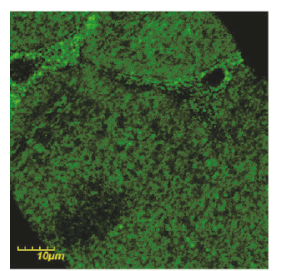

(g)

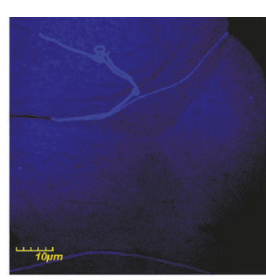

(b)

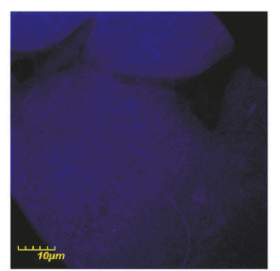

(h)

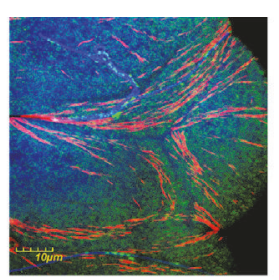

(c)

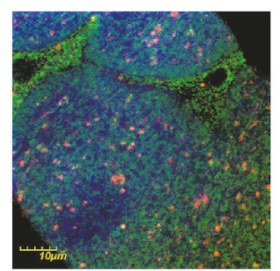

(i)

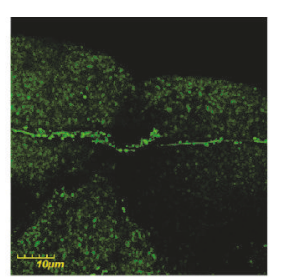

(d)

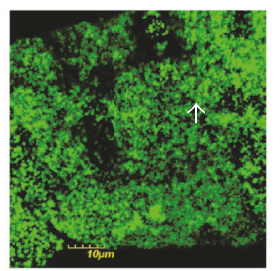

(j)

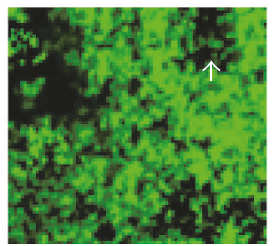

(m)

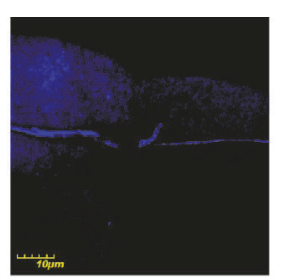

(e)

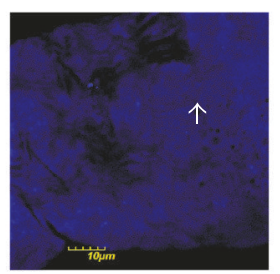

(k)

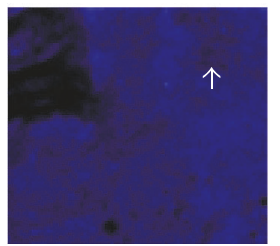

(n)

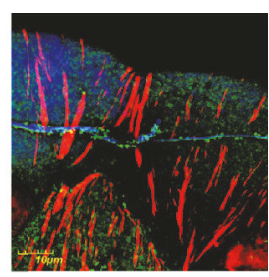

(f)

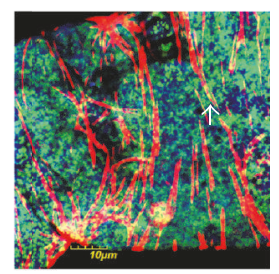

(1)

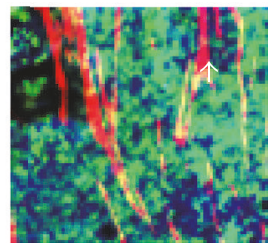

(o)

Figure 2: Protein-HNE adducts in Malpighian epithelium. Color code: mitochondria, green; protein-HNE adducts, blue; actin, red. (a) Mitochondria of wild-type flies incubated at $20^{\circ} \mathrm{C}$, (b) protein-HNE adducts, and (c) merge. ((d), (e), (f)) Wild-type flies incubated at $29^{\circ} \mathrm{C}$, shown in the same order. ((g)-(i)) Mutant flies, incubated at $20^{\circ} \mathrm{C}$, and $((\mathrm{j})-(\mathrm{l}))$ mutant flies, incubated at $29^{\circ} \mathrm{C}$. The order of photomicrographs is as in upper row. Note mitochondrial fusion in ( $\mathrm{j}$ ) and actin stress fibers in (l). White arrow in (j), (k), and (l) showing the portion displayed in higher magnification in (m), (n), and (o). White arrow in $(\mathrm{m}),(\mathrm{n})$, and (o): point regions with no/few mitochondria and the lack of staining, demonstrating localization of alkylated proteins to mitochondria indirectly.

and apparent perinuclear accumulation in the Malpighian epithelial cells in the mutants at permissive conditions $\left(20^{\circ} \mathrm{C}\right)$ (Figures $3(\mathrm{e})$ and $3(\mathrm{f})$ ). This staining pattern was amplified upon shift to restrictive temperature $\left(29^{\circ} \mathrm{C}\right)$ and the HNE-conjugated proteins appeared within the cytoplasmic membrane indicating direct membrane damage by lipid peroxidation (Figures $3(\mathrm{~g})$ and $3(\mathrm{~h})$ ). In the control flies the cytoplasmic membrane remained intact and protein-HNE adducts appeared in the vicinity of the membrane at both permissive and restrictive conditions (Figures 3(a)-3(d)).

3.4. Cell Degeneration Detected by TUNEL-Positivity. The epithelial cells of the Malpighian tubules proved to be TUNEL-positive in mutants at $29^{\circ} \mathrm{C}$ (Figures $4(\mathrm{~d})-4(\mathrm{f})$ ), but not at $20^{\circ} \mathrm{C}$ (Figures $4(\mathrm{a})-4(\mathrm{c})$ ). These observations further supported our earlier observations of cell death affecting multiple tissues in col4al mutants.

\section{Discussion}

Drosophila models provide useful tools for determining the pathomechanistic details, functional alterations, and some of the genotype-phenotype correlations of human monogenic disorders [19] including mutations associated with disorders of the kidneys as some of the human genes known to be associated with inherited nephrotic syndromes play conserved roles in renal functions from flies to humans [20]. There are nephrotic manifestations of human COL4A1 mutations of the Hereditary Angiopathy, Nephropathy, Aneurysms, and Muscle Cramps (HANAC) syndrome [21] and recent research revealed glomerular hyperpermeability and adult onset glomerulocystic kidney disease in association with COL4A1 mutations [4]. Some of the mechanistic elements in context of type IV collagen mutations, such as oxidative stress, have also been demonstrated [22]. However, evidence for chronic inflammation and posttranslational protein modifications are scarce and so far demonstrated only in Drosophila col4al mutants $[7,16]$.

Mitochondrial fusion occurs under situations of cellular stress. Merging of the contents of partially damaged mitochondria is interpreted as a complementation mechanism rescuing impaired organelles and function [23]. Our prior results demonstrated signs of cellular stress in the form of actin stress fibers in the Malpighian epithelial cells of col4al mutant Drosophila [16]. Results of the current study show that mutation-associated stress induced mitochondrial hyperfusion also occurs under restrictive condition with the enlarged organelles unevenly distributed within cells resulting either 


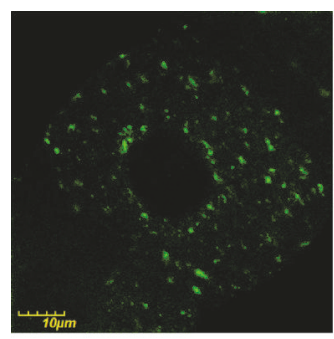

(a)

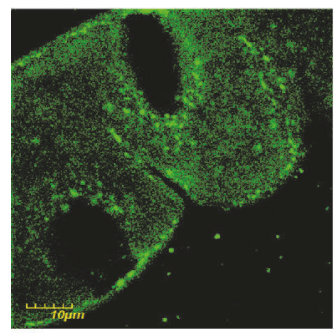

(e)

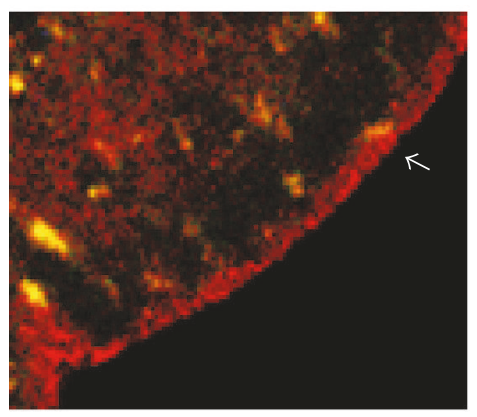

(i)

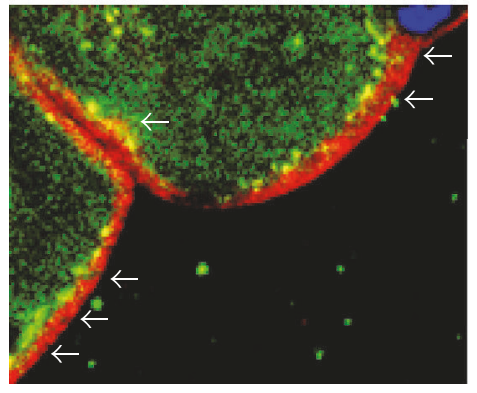

(j)

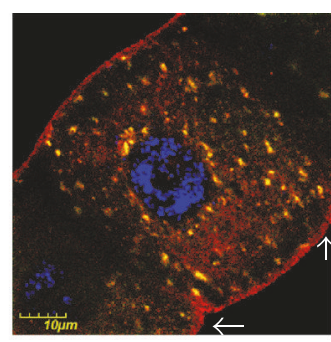

(b)

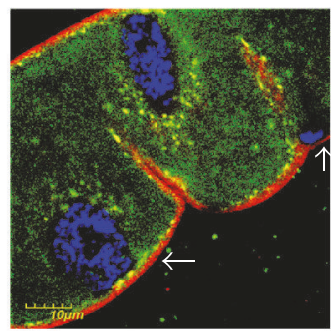

(f)

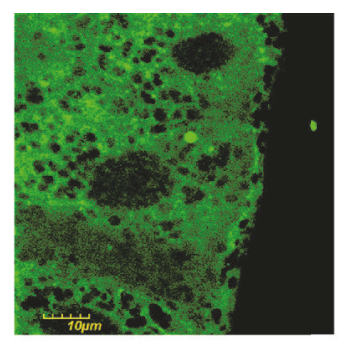

(c)

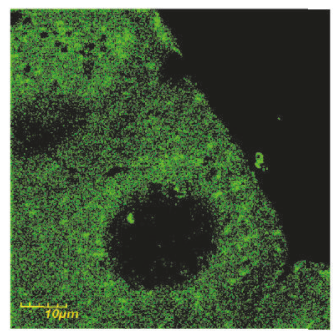

(g)

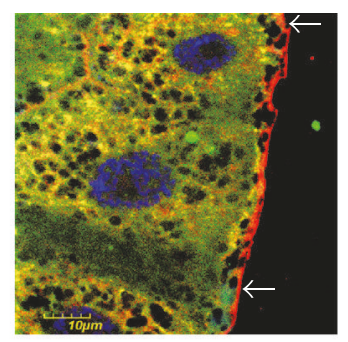

(d)

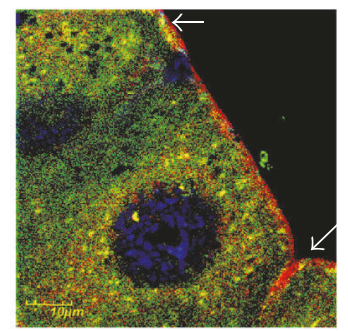

(h)

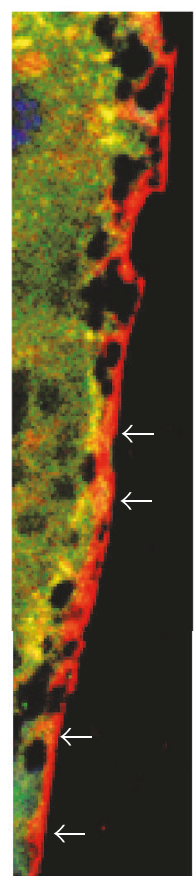

(k)

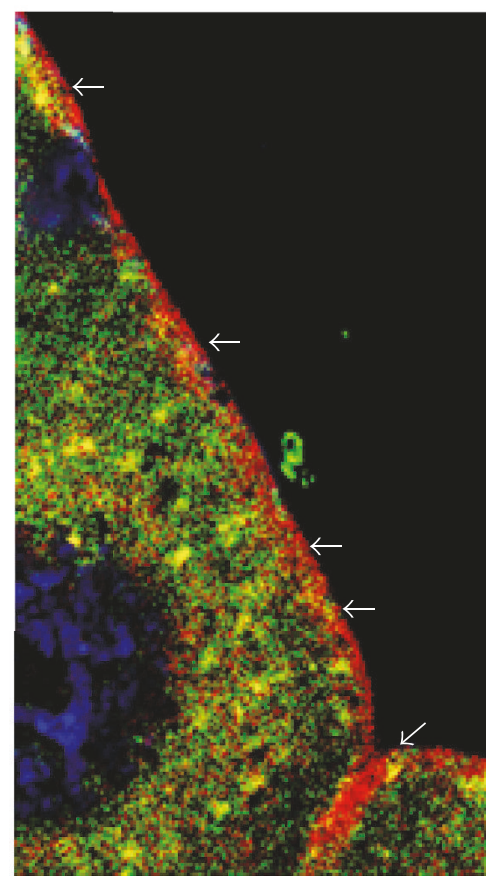

(1)

Figure 3: Cytoplasmic membrane-associated HNE-modified proteins in mutants. Color code: protein-HNE adducts, green; cytoplasmic membrane red; nuclei, blue. (a) Protein-HNE adducts in wild-type flies incubated at $20^{\circ} \mathrm{C}$. (b) Overlay with membrane staining. (c) ProteinHNE adducts in wild-type flies incubated at $29^{\circ} \mathrm{C}$. (d) Merged with membrane staining. ((e), (f), (g), (h)) Representative mutant, incubated at 20 or $29^{\circ} \mathrm{C}$ presented in the same order as in upper row. White arrows in (b), (d), (f), and (h). Regions presented in higher magnification in (i), (j), (k), and (l), respectively. White arrows in (i), (j), (k), and (l) show association of the cytoplasmic membrane with alkylated proteins. Note the notorious infiltration of HNE-modified proteins into the membrane in (l), which occurs at a less extent in (k). The membrane of wild-type animals is free of alkylated proteins (i), while they associate closely with the membrane in the mutant (j), incubated at permissive temperature. 


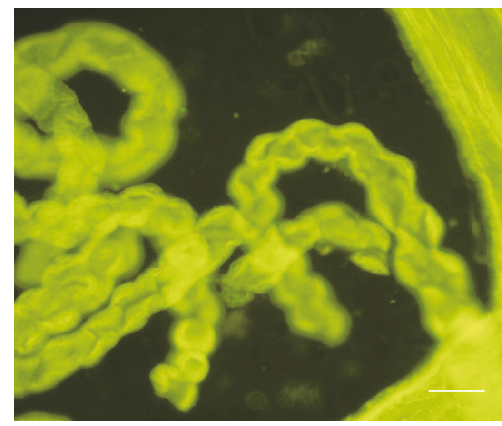

(a)

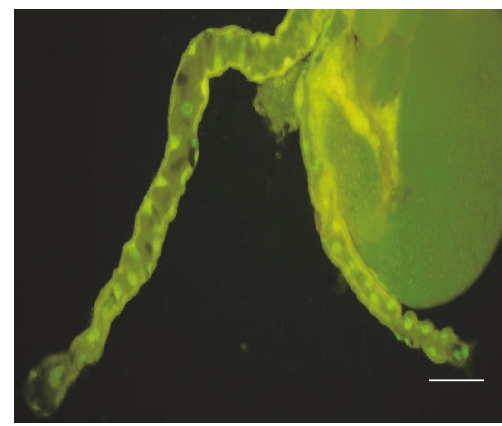

(d)

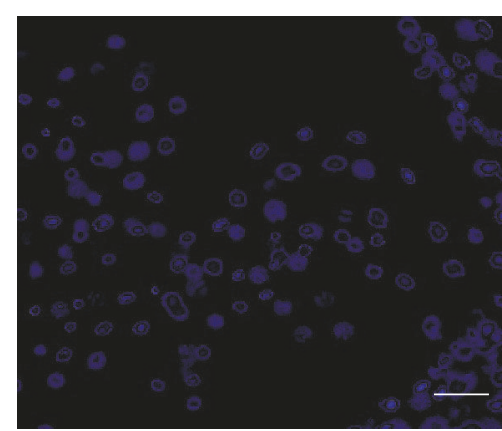

(b)

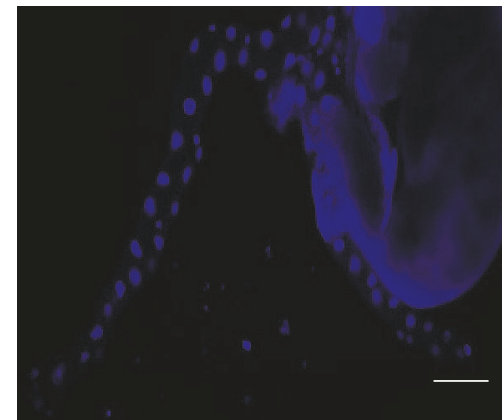

(e)

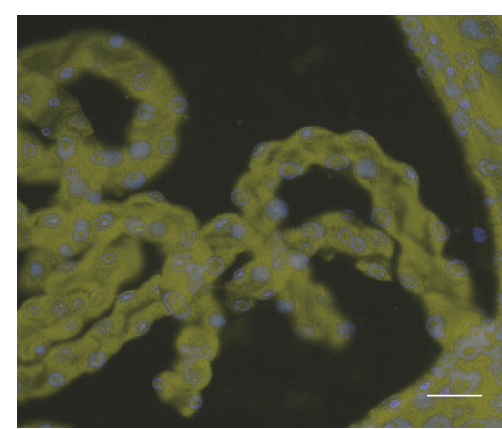

(c)

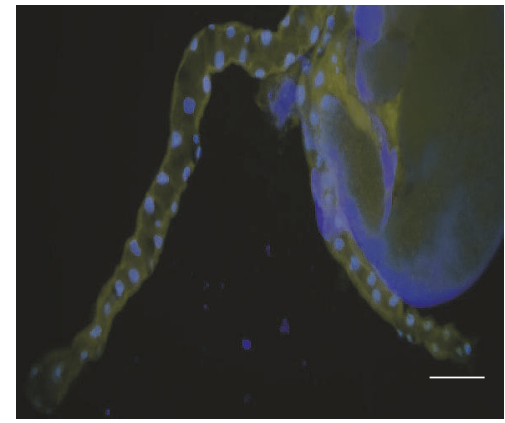

(f)

FIGURE 4: Fluorescence micrographs demonstrating TUNEL-positivity in Malpighian tubules. (a) TUNEL-staining of a Malpighian tubule of col4a1 ${ }^{+/-}$L3-larva incubated at $20^{\circ} \mathrm{C}$; (b) DAPI-staining; (c) merge, tubules appearing TUNEL-negative. (d) TUNEL-positive Malpighian tubule of a col4al ${ }^{+/-}$L3-larva incubated at $29^{\circ} \mathrm{C}$; (d) DAPI; (e) merge. Scale bars: (a)-(c) $50 \mu \mathrm{m}$, (d)-(f) $100 \mu \mathrm{m}$.

in areas apparently lacking mitochondria or in organelleenriched areas. A further consequence of col4al mutation is the accumulation of nitrated and alkylated proteins in the mutants that localize to both normal and fused mitochondria.

This observation indicates a peroxynitrite-mediated nitrosative stress in col4al mutants that produce peroxynitrite at higher concentration [7]. We thus suggest that the elevated peroxynitrite level likely causes excess protein tyrosine nitration; however, this reaction does not deplete peroxynitrite in col4a1 mutants. Indeed, the still available peroxynitrite can initiate membrane damage by lipid peroxidation producing HNE, which in turn alkylates proteins by the mechanism of Michael addiction [8]. Direct association of alkylated proteins with the epithelial cell membrane of mutant Malpighian tubules supports this scenario. Furthermore, the mutationinduced stress directs the epithelia towards degeneration as demonstrated by the TUNEL-positivity of the nuclei.

The data presented here strongly suggest a central role for peroxynitrite in col4a1-associate defects. In wild-type animals and under physiological conditions, the nitrosoperoxycarbonate pathway is the preferential reaction of peroxynitrite, as the main decay products of nitrosoperoxycarbonate, nitrate anion, and carbon dioxide do not exert protein or membrane modification effects (Figure 5) [13]. In mutants, however, peroxynitrite is present above physiological concentrations, and it produces excess protein tyrosine nitration and forms
HNE leading to protein alkylation, lipid peroxidation, membrane damage, aberrant mitochondria, epithelial cell death, and Malpighian tubule dysfunction.

\section{Conclusions}

Drosophila with col4al mutation synthesize peroxynitrite as a part of their stress response above physiological concentrations. The excess peroxynitrite triggers heavy protein tyrosine nitration and protein alkylation adversely affecting protein function; it also initiates membrane lipid peroxidation and mitochondrial fusion. In control animals, these posttranslational protein modifications remain at physiological levels by utilizing the nitrosoperoxycarbonate pathway to neutralize peroxynitrite. We suggest that the posttranslational protein modifications detected in the col4al mutant Drosophila model are integral parts of col4al-associated pathology and represent pathomechanistic details that have not yet been addressed in human or mouse COL $4 A 1$ mutants.

\section{Conflicts of Interest}

The authors declare that there are no conflicts of interest regarding the publication of this paper. 


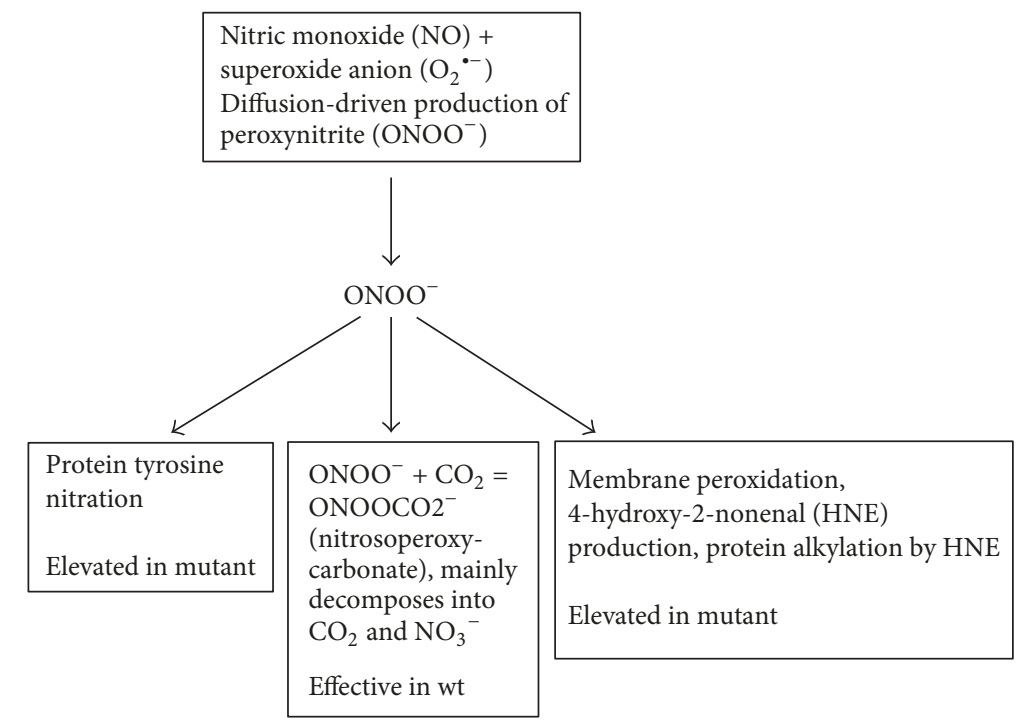

Figure 5: Schematic representation of peroxynitrite effects in wild-type flies shifting towards the neutralizing nitrosoperoxycarbonate pathway and in col4al mutants towards protein nitration and alkylation involving membrane peroxidation.

\section{Acknowledgments}

This research was supported by the Hungarian Scientific Research Fund OTKA, Contract no. NN 108283 to Mátyás Mink, and by the New National Excellence Program, Contract no. UNKP-17-3-I-SZTE-35 to András A. Kiss.

\section{References}

[1] A. Pozzi, P. D. Yurchenco, and R. V. Iozzo, "The nature and biology of basement membranes," Matrix Biology, vol. 57-58, pp. 1-11, 2017.

[2] M. Jeanne and D. B. Gould, "Genotype-phenotype correlations in pathology caused by collagen type IV alpha 1 and 2 mutations," Matrix Biology, vol. 57-58, pp. 29-44, 2017.

[3] D. S. Kuo, C. Labelle-Dumais, and D. B. Gould, "COL4A1 and COL4A2 mutations and disease: insights into pathogenic mechanisms and potential therapeutic targets," Human Molecular Genetics, vol. 21, no. 1, pp. R97-R110, 2012.

[4] Z. Chen, T. Migeon, M.-C. Verpont et al., "HANAC Syndrome Col4al Mutation Causes Neonate Glomerular Hyperpermeability and Adult Glomerulocystic Kidney Disease," Journal of the American Society of Nephrology: JASN, vol. 27, no. 4, pp. 10421054, 2016.

[5] I. Kelemen-Valkony, M. Kiss, J. Csiha et al., "Drosophila basement membrane collagen col4al mutations cause severe myopathy," Matrix Biology, vol. 31, no. 1, pp. 29-37, 2012.

[6] I. Kelemen-Valkony, M. Kiss, K. Csiszár, and M. Mink, Inherited Myopathies, Nova Publishers, New York, NY, USA, 2012.

[7] M. Kiss, A. A. Kiss, M. Radics et al., "Drosophila type IV collagen mutation associates with immune system activation and intestinal dysfunction," Matrix Biology, vol. 49, pp. 120-131, 2016.

[8] F. J. Schopfer, P. R. S. Baker, and B. A. Freeman, "NO-dependent protein nitration: A cell signaling event or an oxidative inflammatory response?" Trends in Biochemical Sciences, vol. 28, no. 12, pp. 646-654, 2003.
[9] A. Denicola and R. Radi, "Peroxynitrite and drug-dependent toxicity," Toxicology, vol. 208, no. 2, pp. 273-288, 2005.

[10] S. N. A. Hussain, G. Matar, E. Barreiro, M. Florian, M. Divangahi, and T. Vassilakopoulos, "Modifications of proteins by 4hydroxy-2-nonenal in the ventilatory muscles of rats," American Journal of Physiology-Lung Cellular and Molecular Physiology, vol. 290, no. 5, pp. L996-L1003, 2006.

[11] S. Dalleau, M. Baradat, F. Guéraud, and L. Huc, "Cell death and diseases related to oxidative stress: 4-hydroxynonenal (HNE) in the balance," Cell Death \& Differentiation, vol. 20, no. 12, pp. 1615-1630, 2013.

[12] G. Juric-Sekhar, K. Zarkovic, G. Waeg, A. Cipak, and N. Zarkovic, "Distribution of 4-hydroxynonenal-protein conjugates as a marker of lipid peroxidation and parameter of malignancy in astrocytic and ependymal tumors of the brain," TUMORI, vol. 95, no. 6, pp. 762-768, 2009.

[13] K. M. Nash, A. Rockenbauer, and F. A. Villamena, "Reactive nitrogen species reactivities with nitrones: Theoretical and experimental studies," Chemical Research in Toxicology, vol. 25, no. 8, pp. 1581-1597, 2012.

[14] J. A. T. Dow and M. F. Romero, "Drosophila provides rapid modeling of renal development, function, and disease," American Journal of Physiology-Renal Physiology, vol. 299, no. 6, pp. F1237-F1244, 2010.

[15] K. W. Beyenbach and P. L.-F. Liu, "Mechanism of fluid secretion common to aglomerular and glomerular kidneys," Kidney International, vol. 49, no. 6, pp. 1543-1548, 1996.

[16] A. A. Kiss, N. Popovics, G. Szabó, K. Csiszár, and M. Mink, "Altered stress fibers and integrin expression in the Malpighian epithelium of Drosophila type IV collagen mutants," Data in Brief, vol. 7, pp. 868-872, 2016.

[17] D. R. LaJeunesse, S. M. Buckner, J. Lake, C. Na, A. Pirt, and K. Fromson, "Three new Drosophila markers of intracellular membranes," BioTechniques, vol. 36, no. 5, pp. 784-790, 2004.

[18] R. Radi, "Nitric oxide, oxidants, and protein tyrosine nitration," Proceedings of the National Acadamy of Sciences of the United States of America, vol. 101, no. 12, pp. 4003-4008, 2004. 
[19] S. Chien, L. T. Reiter, E. Bier, and M. Gribskov, "Homophila: Human disease gene cognates in Drosophila," Nucleic Acids Research, vol. 30, no. 1, pp. 149-151, 2002.

[20] Y. Fu, J.-Y. Zhu, A. Richman et al., "A Drosophila model system to assess the function of human monogenic podocyte mutations that cause nephrotic syndrome," Human Molecular Genetics, vol. 26, no. 4, pp. 768-780, 2017.

[21] E. Plaisier, O. Gribouval, S. Alamowitch et al., "COL4A1 mutations and hereditary angiopathy, nephropathy, aneurysms, and muscle cramps," The New England Journal of Medicine, vol. 357, no. 26, pp. 2687-2695, 2007.

[22] Y. Weng, D. J. Dilworth, R. T. Libby, S. W. John, and D. B. Gould, "Mutant COL4A1 triggers oxidative stress in a genetic model of AMD," Matrix Biology, vol. 27, p. 39, 2008.

[23] R. J. Youle and A. M. van der Bliek, "Mitochondrial fission, fusion, and stress," Science, vol. 337, no. 6098, pp. 1062-1065, 2012. 


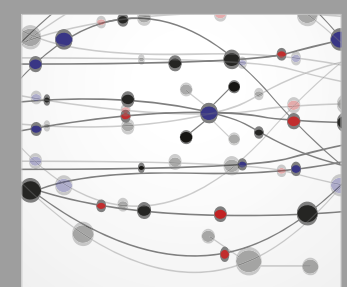

The Scientific World Journal
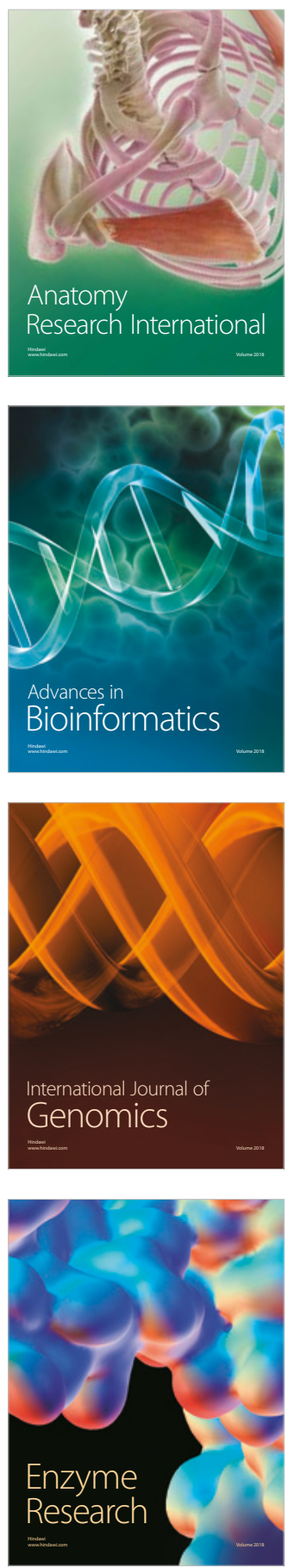
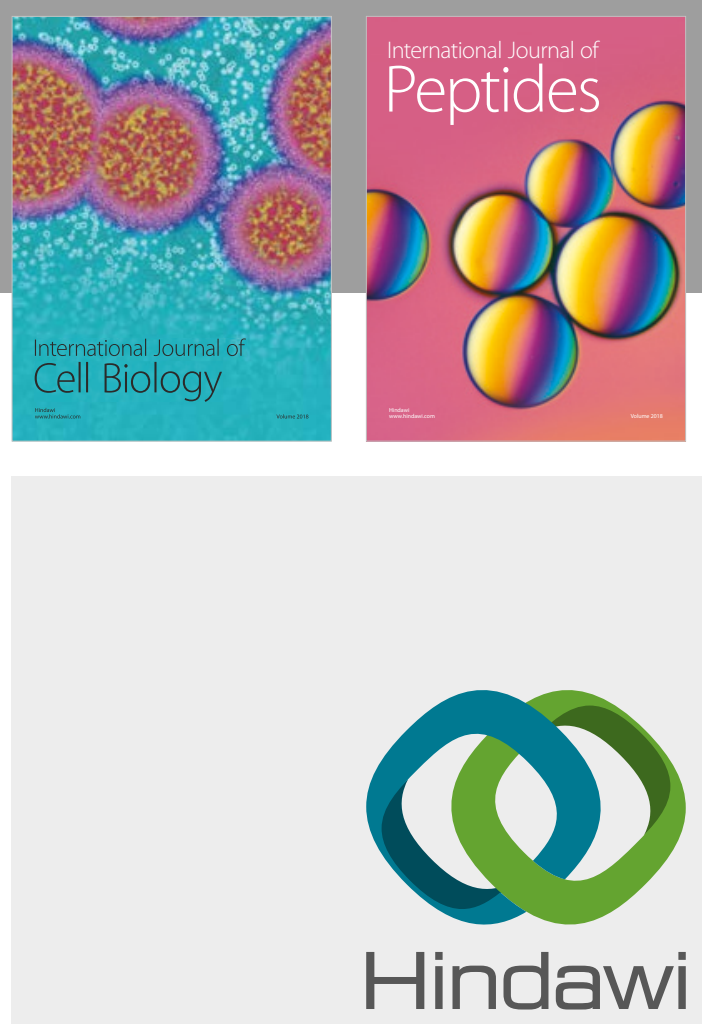

Submit your manuscripts at

www.hindawi.com
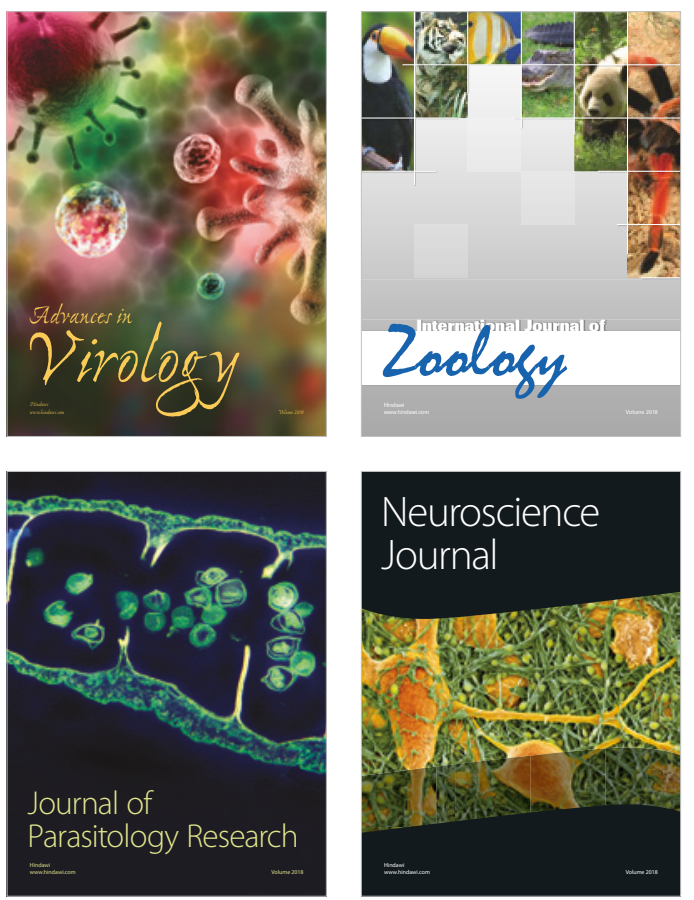
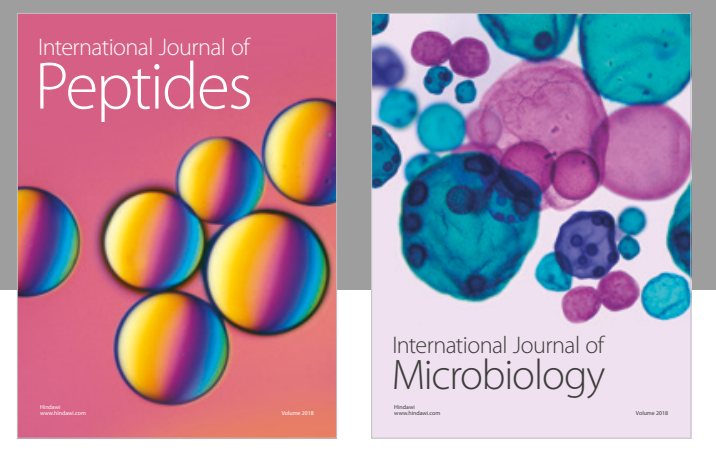

nternational Journal of Microbiology
Journal of
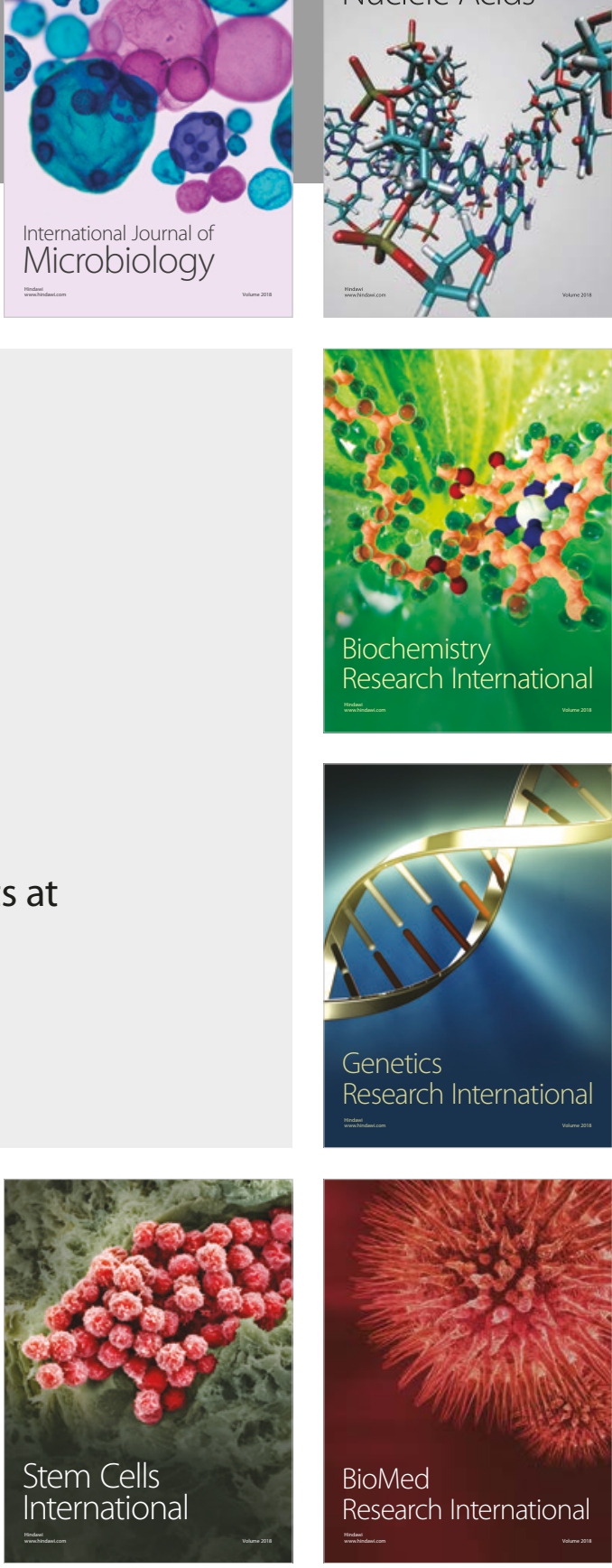
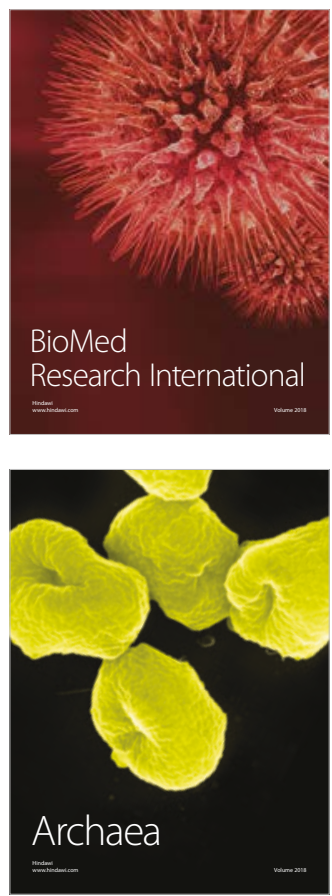